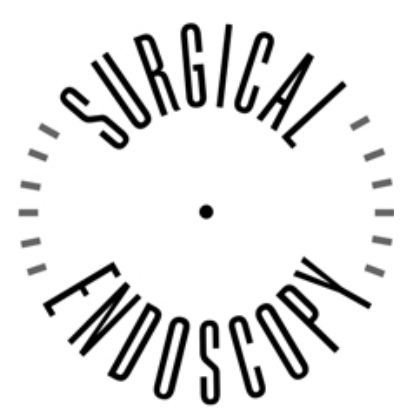

and Other Interventional Techniques

\title{
Laparoscopic radiofrequency ablation and hepatic artery infusion pump placement in the evolving treatment of colorectal hepatic metastases
}

\author{
J. Cheng, R. E. Glasgow, R. W. O'Rourke, L. L. Swanstrom, P. D. Hansen
}

[Surg Endosc (2003) 17: 61-67, DOI: 10.1007/s00464-002-8821-y]

Page 64, left column of Table 3 , line 10 from the top of the column, should read " $\geq 3$ ".

Page 64, left column of Table 3, line 17 from the top of the column, should read " $\geq 4$ ".

In addition, several lines of text, which were correct Online First, were missing in the printed version of the article because of a digital conversion error. The following text should replace the $12^{\text {th }}$ line from the top of text in the left column on page 65 (beneath Table 4 and Fig. 3):

$(p=0.011)($ Fig. 3).

\section{Discussion}

The limited applicability of surgical resection in the treatment of patients with colorectal liver metastases has led surgeons to seek innovative alternatives. Ablative technologies are rapidly being recognized for their potential advantages. The ability to destroy tumor but preserve vital hepatic parenchyma and therefore hepatic reserve may substantially increase the pool of patients who are candidates for treatment. Similarly, the introduction of minimally invasive surgical approaches, which allow staging and aggressive treatment while limiting the impact on the patient's quality of life, may further extend the pool. Finally, there has been a resurgence of interest in the utilization of HAIP chemotherapy due to several recent articles demonstrating a survival benefit $[16,20]$. We have recently published details of a safe and effective laparoscopic approach to the placement of HAIP that may reduce associated surgical morbidity [28]. Using a combination of these techniques seems to make sense intuitively, but there are currently very limited data and even fewer guidelines regarding a rational approach to patient selection and management in these cases.

We have reviewed the data for our first 45 patients treated using LRFA and/or LHAIP placement for the treatment of isolated colorectal metastases to the liver. The value of these data is necessarily compromised by the introduction of changing technologies (e.g., three different RFA generators and five different RFA probes) during the 5-year period described. Some degree of stability was achieved during the final 4-year period (19972001) following the introduction of 150-200-W generators and $5-\mathrm{cm}$ ablation probes. Further variability was introduced by an evolving understanding of the appropriate use of these technologies and improved technical skills as we moved beyond the learning curve. Finally, a direct comparison of the three different treatment groups is provided; however, patients were selected to be in their relative group by their clinical presentation. We are not recommending one treatment regimen over another. Instead, our goal is to describe our treatment selection process and review the short-term outcomes.

RFA is now gaining recognition as 